九州大学学術情報リポジトリ

Kyushu University Institutional Repository

\title{
Markov Chains with a Transition Possibility Measure and Fuzzy Dynamic Programming
}

Yoshida, Yuji

Faculty of Economics, Kitakyushu University

http://hdl. handle. net/2324/3185

出版情報 : RIFIS Technical Report. 88，1994-05-09. Research Institute of Fundamental Information Science, Kyushu University バージョン:

権利関係 : 


\section{RIFIS Technical Report}

Markov Chains with a Transition Possibility Measure

and Fuzzy Dynamic Programming

Yuji Yoshida

May 9, 1994

Research Institute of Fundamental Information Science

Kyushu University 33

Fukuoka 812, Japan 


\title{
MARKOV CHAINS WITH A TRANSITION POSSIBILITY MEASURE AND FUZZY DYNAMIC PROGRAMMING
}

\author{
Yuji YOSHIDA \\ Faculty of Economics, Kitakyushu University, \\ Kitagata, Kokuraminami, Kitakyushu 802, Japan
}

\begin{abstract}
This paper constructs a Markov fuzzy process, which represents transitions of grades of fuzzy sets, with a transition possibility measure and a general state space. We analyse Snell's optimal stopping problem for the process and we apply the results to solve fuzzy dynamic programming with optimal stopping times and with general state spaces and action spaces under fuzzy transitions.
\end{abstract}

Keyword : Markov fuzzy system; fuzzy dynamic programming; Snell's optimal stopping problem.

\section{Introduction}

Bellman and Zadeh [1] has first studied a fuzzy decision process. The problem with optimal stopping times is studied by Kacprzyk [5] and Stein [12]. Esogbue and Bellman [4] developed various kinds of fuzzy dynamic programming with finite state spaces and finite action spaces. Especially, [4, Sect.5] studied fuzzy dynamic programming with terminal times and [4, Sect.7] studied finite-stage fuzzy dynamic programming under a fuzzy transition. These fuzzy dynamic programmings are different from the classical dynamic programming, in the point of using grades of fuzzy sets instead of reward functions. Therefore the systems for fuzzy dynamic programmings are generally non-linear. This paper deals with fuzzy dynamic programming with optimal stopping times and with general state spaces and action spaces.

Kurano et al. [6] and Yoshida et al. [14] studied dynamic fuzzy systems, which are transit as follows :

Let $S$ be a metric space. We write a fuzzy set on $S$ by its membership function $\tilde{s}: S \mapsto[0,1]$. Let $\mathcal{F}^{0}(S)$ be the set of all fuzzy sets on $S$, which are upper semicontinuous and have a compact support. For any $\tilde{s} \in \mathcal{F}^{0}(S)$, we define

$$
\tilde{q}(\tilde{s})(x):=\sup _{y \in S}\{\tilde{q}(x, y) \wedge \tilde{s}(y)\}, x \in S
$$

and

$$
\tilde{q}^{0}(\tilde{s}):=\tilde{s} \quad \text { and } \quad \tilde{q}^{n}(\tilde{s}):=\tilde{q}\left(\hat{q}^{n-1}(\tilde{s})\right), \quad n=1,2, \cdots .
$$

Then $\tilde{q}^{n}(\tilde{s})(x)$ is called a transition possibility from a point $x$ to a fuzzy set $\tilde{s}$ at $n^{\text {th }}$-step ([14]). 
From the reason explained in the latter part of this section, we need to define the transition possibility for a certain class of fuzzy sets containing $\mathcal{F}^{0}(E)$. We consider a fuzzy expectation as an extension of the transition possibility for the class.

Expectations by fuzzy measures are studied by Sugeno [13]. However, when we deal with uncountable state spaces, it is known that there is an essential difference between fuzzy measures and possibility measures (see Puri and Ralescu [9]). Cooman and Kerre [3] studied the expectations by possibility measures. However [3] does not take account of the measurability of expectations. This paper introduces a fuzzy expectation of measurable membership functions by a possibility measure induced by fuzzy relations, preserving the measurability. We also introduce a path structure for a dynamic fuzzy system with a possibility measure induced by fuzzy relations and construct a non-linear Markov fuzzy process representing transitions of grades of fuzzy sets. This enables us to deal with stopping times depending on paths analytically.

We consider Snell's optimal stopping problem (Neveu [7, Sect.VI-2]) for the Markov fuzzy system. The problem is to find paths and stopping times maximizing the grade of fuzzy sets. The aim of this paper is to study fuzzy dynamic programming with general state spaces and general action spaces and with optimal stopping times depending on paths, using the solutions of Snell's problem for the Markov fuzzy system. We derive an optimality equation for the problem and we give an optimal stopping time bounded on an optimal path.

First of all, we consider about a class of fuzzy sets, containing $\mathcal{F}^{0}(S)$, which admits stopping times. We put the collection of all closed subsets of $S$ by $\mathcal{C}(S)$ and the Borel- $\sigma$ field of $S$ by $\mathcal{B}(S)$. We write an ordinary set $A(\subset S)$ by its indicator function $1_{A}: S \mapsto$ $\{0,1\}$. The $\alpha$-cut $\tilde{s}_{\alpha}$ of a fuzzy set $\tilde{s}$ is written by

$$
\tilde{s}_{\alpha}:=\{x \in S \mid \tilde{s}(x) \geq \alpha\}(\alpha \in(0,1]) \quad \text { and } \quad \tilde{s}_{0}:=\operatorname{cl}\{x \in S \mid \tilde{s}(x)>0\},
$$

where cl denotes the closure of a set. The fuzzy set $\tilde{s} \in \mathcal{F}^{0}(S)$ is characterized by its $\alpha$-cuts :

(C.i) $\tilde{s}_{0}$ is compact;

(C.ii) $\tilde{s}_{\alpha} \in \mathcal{C}(S)$ for $\alpha \in[0,1]$;

(C.iii) $\bigcap_{\alpha^{\prime}<\alpha} \tilde{s}_{\alpha^{\prime}}=\tilde{s}_{\alpha}$ for $\alpha \in(0,1]$.

What kind of measurable fuzzy sets can we define stopping times for ? For an answer to this question, we here consider about an essential difficulty in optimal stopping problems for fuzzy systems (more generally, fuzzy decision processes) with general state spaces, through the following one-stage example:

Let $A_{1}$ and $A_{2}$ be subsets of a state space $S$. We suppose that a return $Z_{1}$ is given if we stop the process and a return $Z_{2}$ is given if not to stop. We consider a decision 
that we stop when the present state is in $A_{1}$ and we do not stop when the state is in $A_{2}$. The return obtained by the decision is given by

$$
Z:= \begin{cases}Z_{1} & \text { on } A_{1} \\ Z_{2} & \text { on } A_{2}\end{cases}
$$

Then $A_{1}$ and $A_{2}$ must have the following property :

$$
A_{1} \cap A_{2}=\emptyset \quad \text { and } \quad A_{1} \cup A_{2}=S \text {. }
$$

However, when we construct a fuzzy system for fuzzy sets of $\mathcal{F}^{0}(S), A_{1}$ and $A_{2}$ must be closed because of (C.ii) and $Z_{1}, Z_{2}, Z \in \mathcal{F}^{0}(S)$. This fact contradicts (0.4) when $S$ is a general metric space. Therefore this paper extends $\mathcal{F}^{0}(S)$ to some class of measurable fuzzy sets admitting stopping times. In order to preserve a measurability of fuzzy sets regarding a fuzzy expectation by a possibility measure induced by fuzzy relations, we find, in Section 1, that the measurability must be preserved regarding projections from a product space. For example, Borel measurability is not proper since a projection of a Borel set in a product space is in general not Borel and but analytic (see Bertsekas and Shreve [2, Sect.7.6]). The collection of analytic sets is not also proper. Because analytic sets are in general not Borel and but universally measurable. However a $\sigma$-field of universally measurable sets can not be represented by a completion of Borel sets by possibility measures. We adopt the collection of $F_{\sigma}$-sets (see Parthasarathy [8, Def.1.1]) and construct a Markov fuzzy system with a transition possibility measure. Using the results, we solve fuzzy dynamic programming under a fuzzy transition and Snell's optimal stopping problem for the Markov fuzzy system.

In Section 1 we introduce a path structure like the theory of stochastic processes. We also introduce a fuzzy expectation by a transition possibility measure and an admissible class of stopping times. In Section 2 we prove a strong Markov property for the stopping times and construct, as a fuzzy process, a Markov fuzzy system under the possibility measure. In Section 3 we extend the expectation in the preparation for Section 4. In Section 4 we solve Snell's optimal stopping problem for the Markov fuzzy system. In Section 5 we apply the results to fuzzy dynamic programming with stopping times depending on paths under the fuzzy transition.

\section{Fuzzy expectations and stopping times}

For a metric space $S$, we put the collection of $F_{\sigma}$-sets by $\mathcal{E}(S):=\left\{A \mid A=\bigcup_{n=0}^{\infty} C_{n}, C_{n} \in\right.$ $\mathcal{C}(S)(n=0,1,2, \cdots)\}$, where $\mathcal{C}(S)$ is defined in Introduction. $\mathcal{F}(S)$ denotes the set of all fuzzy sets on $S$ satisfying the following conditions (E.i) - (E.iii) :

(E.i) $\tilde{s}_{0}$ is compact;

(E.ii) $\tilde{s}_{\alpha} \in \mathcal{E}(S)$ for $\alpha \in[0,1]$ 
(E.iii) $\bigcap_{\alpha^{\prime}<\alpha} \tilde{s}_{\alpha^{\prime}}=\tilde{s}_{\alpha}$ for $\alpha \in(0,1]$.

We note that $\mathcal{C}(S) \subset \mathcal{E}(S) \subset \mathcal{B}(S)$ and $\mathcal{F}^{0}(S) \subset \mathcal{F}(S)$.

Lemma 1.1. The collection of $F_{\sigma}$-sets, $\mathcal{E}(S)$, has the following properties :

(i) $\mathcal{E}(S)$ is closed under finite intersections and countable unions;

(ii) Let $S_{1}$ and $S_{2}$ be metric spaces. Let $A \in \mathcal{E}\left(S_{1} \times S_{2}\right)$. Then its projection from $S_{1} \times S_{2}$ to $S_{1}$, $\operatorname{proj}_{S_{1}}(A)$, has a property : $\operatorname{proj}_{S_{1}}(A) \in \mathcal{E}\left(S_{1}\right)$.

Proof. (i) Let $\left\{A_{m}\right\}_{m=0}^{\infty} \subset \mathcal{E}(S)$. Then there exists $C_{m, n} \in \mathcal{C}(S)(m, n=0,1,2, \cdots)$ such that $A_{m}=\bigcup_{n=0}^{\infty} C_{m, n}$ for all $m$. Therefore $\bigcup_{m=0}^{\infty} A_{m}=\bigcup_{m, n=0}^{\infty} C_{m, n} \in \mathcal{E}(S)$.

Next let $\left\{A_{m}\right\}_{m=0}^{l} \subset \mathcal{E}(S)$ for some $l=0,1,2, \cdots$. Then there exists $C_{m, n} \in \mathcal{C}(S)(m=$ $0,1,2, \cdots, l ; n=0,1,2, \cdots)$ such that $A_{m}=\bigcup_{n=0}^{\infty} C_{m, n}$ for all $m=0,1,2, \cdots, l$. Here we may assume that $\left\{C_{m, n}\right\}_{n=0}^{\infty} \subset \mathcal{C}(S)$ is non-decreasing, considering $C_{m, n}^{\prime}:=\bigcup_{k=0}^{n} C_{m, k} \in$ $\mathcal{C}(S)$. Now we show $\bigcap_{m=0}^{l} \bigcup_{n=0}^{\infty} C_{m, n}=\bigcup_{n=0}^{\infty} \bigcap_{m=0}^{l} C_{m ; n}$. Let $x \in \bigcap_{m=0}^{l} \bigcup_{n=0}^{\infty} C_{m, n}$. Then for each $m$ there exists $n_{m}=0,1,2, \cdots$ satisfying $x \in C_{m, n}$ for all $n \geq n_{m}$, since $\left\{C_{m, n}\right\}_{n=0}^{\infty}$ is non-decreasing. Put $n^{*}:=\max _{m=0,1,2, \cdots, l} n_{m}(<\infty)$. Then $x \in \bigcap_{m=0}^{l} C_{m, n^{*}} \subset$ $\bigcup_{n=0}^{\infty} \bigcap_{m=0}^{l} C_{m, n}$. Therefore we obtain $\bigcap_{m=0}^{l} \bigcup_{n=0}^{\infty} C_{m, n} \subset \bigcup_{n=0}^{\infty} \bigcap_{m=0}^{l} C_{m, n}$. The reverse inclusion is clear. Thus we get $\bigcap_{m=0}^{l} A_{m}=\bigcap_{m=0}^{l} \bigcup_{n=0}^{\infty} C_{m, n}=\bigcup_{n=0}^{\infty} \bigcap_{m=0}^{l} C_{m, n} \in \mathcal{E}(S)$.

(ii) Let $A \in \mathcal{E}\left(S_{1} \times S_{2}\right)$. Then $A=\bigcup_{n=0}^{\infty} C_{n}$ for some $C_{n} \in \mathcal{C}\left(S_{1} \times S_{2}\right)(n=0,1,2, \cdots)$. We have $\operatorname{proj}_{S_{1}}(A)=\bigcup_{y \in S_{2}}\left\{x \in S_{1} \mid(x, y) \in A\right\}=\bigcup_{y \in S_{2}} \bigcup_{n=0}^{\infty}\left\{x \in S_{1} \mid(x, y) \in C_{n}\right\}=$ $\bigcup_{n=0}^{\infty} \bigcup_{y \in S_{2}}\left\{x \in S_{1} \mid(x, y) \in C_{n}\right\}$. Since $\bigcup_{y \in S_{2}}\left\{x \in S_{1} \mid(x, y) \in C_{n}\right\} \in \mathcal{C}\left(S_{1}\right)$, we obtain $\operatorname{proj}_{S_{1}}(A) \in \mathcal{E}\left(S_{1}\right)$.

The following proposition illustrates a regularity of $\mathcal{E}(S)$ when 1-dimensional case. Let $\mathbf{R}^{1}$ denote the set of all real numbers.

Proposition 1.1. Assume $S:=\mathbf{R}^{1}$. Denote the set of all intervals by

$$
\mathcal{I}(S):=\left\{\text { intervals } I \mid I=(a, b),[a, b],(a, b],[a, b) \text { for } a, b \in \mathbf{R}^{1}(a \leq b)\right\} .
$$

Then $\bigcup_{n=0}^{\infty} I_{n} \in \mathcal{E}(S)$ for $\left\{I_{n}\right\}_{n=0}^{\infty} \subset \mathcal{I}(S)$.

Proof. It is trivial that $[a, b] \in \mathcal{E}(S)$ for $a, b \in \mathbf{R}^{1}(a \leq b)$. Further, from Lemma 1.1(i), $(a, b]=\bigcup_{m=1}^{\infty}[a+1 / m, b] \in \mathbf{R}^{1}(a \leq b)$. Considering about the others similarly, we obtain $\mathcal{I}(S) \subset \mathcal{E}(S)$. Therefore we get this proposition, using Lemma 1.1(i).

Now we construct a fuzzy process with a transition possibility measure. In Section 2 we show the fuzzy process has a strong Markov property. Let a discrete time space $\mathbf{N}:=\{0,1,2,3, \cdots\}$ and $\overline{\mathbf{N}}:=\mathbf{N} \cup\{\infty\}$. Let a state space $E$ be a finite-dimensional Euclidean space. Let $E_{0}, E_{1}, E_{2}, \cdots$ denote copies of $E$. Then the path space is $\Omega:=$ $\prod_{k=0}^{\infty} E_{k}$, which is metrizable by the product topology. We also put $\Omega_{n}:=\prod_{k=n}^{\infty} E_{k}$ for $n \in \mathbf{N}$. An element $\omega=(\omega(0), \omega(1), \omega(2), \cdots) \in \Omega$ is called a sample path. We define a 
$\operatorname{map} X_{n}(\omega):=\omega(n)$ and a shift $\theta_{n}(\omega):=(\omega(n), \omega(n+1), \cdots)$ for $\omega \in \Omega$ and $n \in \mathbf{N}$. We put $\sigma$-fields by $\mathcal{M}_{n}:=\sigma\left(X_{0}, X_{1}, \cdots, X_{n}\right)^{1}$ for $n \in \mathbf{N}$ and $\mathcal{M}:=\sigma\left(\bigcup_{n \in \mathbb{N}} \mathcal{M}_{n}\right)^{2}$. For a sub- $\sigma$-field $\mathcal{N}$ of $\mathcal{M}, \mathcal{F}(\mathcal{N})$ denotes the set of all $\mathcal{N}$-measurable fuzzy sets of $\mathcal{F}(\Omega)$.

Let $\tilde{q}_{n}:=\tilde{q}$ be a time-invariant upper semi-continuous fuzzy relation on $E_{n} \times E_{n+1}$ $(n=0,1,2, \cdots)$ satisfying the following normality condition : For all $n \in \mathbf{N}$,

$$
\sup _{x \in E_{n}} \tilde{q}_{n}(x, y)=1\left(y \in E_{n+1}\right) \text { and } \sup _{y \in E_{n+1}} \tilde{q}_{n}(x, y)=1\left(x \in E_{n}\right) .
$$

We define operations $\bigwedge_{n=0}^{\infty}$ and $\bigvee_{n=0}^{\infty}$ by $\bigwedge_{n=0}^{\infty} h_{n}(\omega):=\inf _{n=0,1,2, \ldots} h_{n}(\omega)$ and $\bigvee_{n=0}^{\infty} h_{n}(\omega):=$ $\sup _{n=0,1,2, \ldots} h_{n}(\omega)$ for $\left\{h_{n}\right\}_{n=0}^{\infty} \subset \mathcal{F}(\mathcal{M})$ and $\omega \in \Omega$. We define $q:=\bigwedge_{n=0}^{\infty} \tilde{q}_{n}\left(X_{n}, X_{n+1}\right) \in$ $\mathcal{F}(\mathcal{M})$ and $P(\Lambda):=\sup _{\omega \in \Lambda} q(\omega)$ for $\Lambda \in \mathcal{M}$. Then $P$ becomes a possibility measure on $\mathcal{M}$ with a density $q$ (see Puri and Ralescu [9]) and has the following properties (P.i) (P.iii) :

(P.i) $P(\emptyset)=0$ and $P(\Omega)=1$;

(P.ii) If $\Lambda, \Gamma \in \mathcal{M}$ satisfy $\Lambda \subset \Gamma$, then $P(\Lambda) \leq P(\Gamma)$;

(P.iii) If $\left\{\Lambda_{n}\right\}_{n=0}^{\infty} \subset \mathcal{M}$, then $P\left(\bigcup_{n=0}^{\infty} \Lambda_{n}\right)=\bigvee_{n=0}^{\infty} P\left(\Lambda_{n}\right)$.

For $h \in \mathcal{F}(\mathcal{M})$, we define a fuzzy expectation by the possibility measure $P$ :

$$
\begin{aligned}
E_{x}(h) & :=f_{\{\omega \in \Omega: \omega(0)=x\}} h(\omega) \mathrm{d} P(\omega) \\
& =\sup _{\omega \in \Omega: \omega(0)=x} h(\omega) \wedge q(\omega) \quad \text { for } x \in E,
\end{aligned}
$$

where $f \mathrm{~d} P$ denotes Sugeno integral and the supremum on the empty set is understood to be 0 (see Sugeno [13], Ralescu and Adams [10]), and $a \wedge b:=\min \{a, b\}$ and $a \vee b$ $:=\max \{a, b\}$ for real numbers $a, b$. For $x \in E$ we also define a possibility measure $P_{x}(\Lambda):=E_{x}\left(1_{\Lambda}\right)(\Lambda \in \mathcal{M})$.

Here we consider the meaning of the fuzzy expectation. From $(0.1)$ and $(0.2)$, for $\tilde{s} \in \mathcal{F}^{0}(E)$ we can easily check

$$
E_{x}\left(\tilde{s}\left(X_{n}\right)\right)=\tilde{q}^{n}(\tilde{s})(x) \quad \text { for } x \in E, n \in \mathbf{N},
$$

which is a possibility of transition from a point $x$ to a fuzzy set $\tilde{s}$ at $n^{\text {th }}$-step. Therefore we can define the transition possibility for the class $\mathcal{F}(E)$, containing $\mathcal{F}^{0}(E)$, by means of the fuzzy expectation $E_{x}\left(\tilde{s}\left(X_{n}\right)\right)$.

Next we take $g=\bigwedge_{k=0}^{n-1} 1_{\left\{X_{k+1} \in \Lambda_{k+1}\right\}} \in \mathcal{F}(\mathcal{M})(n \in \mathbf{N})$. From the definition of the fuzzy expectation, we have

$$
E_{x}(g)=\sup _{\omega \in \Omega} \bigwedge_{k=0}^{n-1} 1_{\left\{X_{k+1} \in \Lambda_{k+1}\right\}}(\omega) \wedge \tilde{q}_{k}\left(X_{k} \omega, X_{k+1} \omega\right) \text { for } x \in E .
$$

\footnotetext{
${ }^{1}$ It denotes the smallest $\sigma$-field on $\Omega$ relative to which $X_{0}, X_{1}, \cdots, X_{n}$ are measurable.

${ }^{2}$ It denotes the smallest $\sigma$-field generated by $\bigcup_{n \in \mathbb{N}} \mathcal{M}_{n}$.
} 
Then we observe a path $\omega$ which is starting from $X_{0} \omega=x$, stays at $X_{1} \omega \in \Lambda_{1}$ at $1^{\text {st }}$. step, stays at $X_{2} \omega \in \Lambda_{2}$ at $2^{\text {nd }}$-step, $\cdots$, and arrives at $X_{n} \omega \in \Lambda_{n}$ at $n^{\text {th }}$-step. (1.2) is a maximum possibility over all these paths $\omega$. The representation (1.2) of the transition possibility by paths enables us to introduce stopping times depending on paths in this section. Therefore the fuzzy expectation is an extension of the transition possibility and admits stopping times depending on paths. We call $\left\{X_{n}\right\}_{n \in \mathrm{N}}$ a fuzzy process with the possibility measure $P$. The fuzzy process $\left\{X_{n}\right\}_{n \in \mathrm{N}}$ transits based on the fuzzy transition in the meanings of $(1.1),(1.2)$, which is corresponding to the transition of the grade of fuzzy sets.

Lemma 1.2. Let $h \in \mathcal{F}(\mathcal{M})$. Then :

(i) the map $x \mapsto E_{x}(h)$ is an element of $\mathcal{F}(E)$;

(ii) $E_{x}(h)=\bigvee_{m=0}^{\infty} E_{x}\left(h \wedge 1_{\Lambda_{m}}\right)$

for $x \in E$ and $\left\{\Lambda_{m}\right\}_{m=0}^{\infty} \subset \mathcal{E}(\Omega)$ such that $\left\{\Lambda_{m}\right\}_{m=0}^{\infty}$ is a partition of $\Omega$.

Proof. (i) Since $h, q \in \mathcal{F}(\mathcal{M})$, from Lemma 1.1(i)(ii), we have

$\left\{x \mid E_{x}(h) \geq \alpha\right\}=\bigcup_{\omega \in \Omega}\{x \mid \omega(0)=x, h(\omega) \wedge q(\omega) \geq \alpha\}=\operatorname{proj}_{E_{1}}\{\omega \mid h(\omega) \wedge q(\omega) \geq \alpha\} \in \mathcal{E}\left(E_{1}\right)$

for $\alpha \in[0,1]$. (ii) can be easily checked from the definition.

Definition. For $\mathcal{M}$-measurable fuzzy sets $h, g: \Omega \mapsto[0,1]$ and $x \in E, h$ and $g$ are called $P_{x}$-equivalent if $h(\omega) \wedge q(\omega)=g(\omega) \wedge q(\omega)$ for all $\omega \in \Omega: \omega(0)=x$. Then we write $h=g$ $P_{x^{-}}$almost surely $\left(P_{x^{-}}\right.$-a.s. $)$. Further we represent $h=g$ a.s. when $h=g P_{x^{-a}}$.s. holds for all $x \in E$.

We introduce another fuzzy expectation.

Definition. Let $h \in \mathcal{F}(\mathcal{M})$ and $n \in \mathbf{N}$. We define a fuzzy expectation of $h$ with a sub- $\sigma$-field $\mathcal{M}_{n}$ by

$$
\begin{aligned}
E\left(h \mid \mathcal{M}_{n}\right)(\omega) & :=f_{\Omega_{n+1}} h \mathrm{~d} P_{\omega, n} \\
& =\sup _{\omega^{\prime} \in \Omega: \omega^{\prime}(k)=\omega(k)(k=0,1, \cdots, n)} h\left(\omega^{\prime}\right) \wedge \bigwedge_{k=n}^{\infty} \tilde{q}_{k}\left(X_{k} \omega^{\prime}, X_{k+1} \omega^{\prime}\right) \quad \text { for } \omega \in \Omega,
\end{aligned}
$$

where

$$
P_{\omega, n}(\Lambda):=\sup _{\omega^{\prime} \in \Lambda: \omega^{\prime}(k)=\omega(k)(k=0,1, \cdots, n)} \bigwedge_{k=n}^{\infty} \tilde{q}_{k}\left(X_{k} \omega^{\prime}, X_{k+1} \omega^{\prime}\right) \quad \text { for } \omega \in \Omega, n \in \mathbf{N} \text { and } \Lambda \in \mathcal{M}
$$

Lemma 1.3. It holds that

$$
\left\{X_{n} \in A\right\} \in \mathcal{E}(\Omega) \text { for all } n \in \mathbf{N} \text { and } A \in \mathcal{E}(E) .
$$


Proof. Let $n \in \mathbf{N}$. If $C$ is a closed subset of $E$, then $\left\{X_{n} \in C\right\}=\prod_{k=0}^{n-1} E_{k} \times C \times \prod_{k=n+1}^{\infty} E_{k}$ is closed in $\Omega$. Therefore for $A=\bigcup_{m=0}^{\infty} C_{m} \in \mathcal{E}(E)\left(C_{m} \in \mathcal{C}(E), m=0,1,2, \cdots\right)$, we have $\left\{X_{n} \in A\right\}=\bigcup_{m=0}^{\infty}\left\{X_{n} \in C_{m}\right\} \in \mathcal{E}(\Omega)$.

The following proposition shows that the fuzzy expectation $E\left(h \mid \mathcal{M}_{n}\right)$ has the properties like the conditional expectations in the probability theory of Neveu [7].

Proposition 1.2. Let $h \in \mathcal{F}(\mathcal{M})$ and $n \in \mathbf{N}$. Then $E\left(h \mid \mathcal{M}_{n}\right)$ is a fuzzy set in $\mathcal{F}\left(\mathcal{M}_{n}\right)$ and is the unique $\mathcal{M}_{n}$-measurable fuzzy set (in the sense of a.s. ) such that

$$
f_{\Lambda} E\left(h \mid \mathcal{M}_{n}\right) d P_{x}=f_{\Lambda} h d P_{x} \quad \text { for all } \Lambda \in \mathcal{M}_{n} \text { and } x \in E .
$$

Proof. For all $\Lambda \in \mathcal{M}_{n}$ and $x \in E$, we have

$$
\begin{aligned}
f_{\Lambda} & E\left(h \mid \mathcal{M}_{n}\right) \mathrm{d} P_{x} \\
= & f_{\Lambda}\left(f_{\Omega_{n}} h \mathrm{~d} P_{\omega, n}\right) \mathrm{d} P_{x}(\omega) \\
= & \sup _{\omega \in \Lambda: \omega(0)=x}\left(\sup _{\omega^{\prime} \in \Omega: \omega^{\prime}(k)=\omega(k)(k=0,1, \cdots, n)} h\left(\omega^{\prime}\right) \wedge \bigwedge_{k=n}^{\infty} \tilde{q}_{k}\left(X_{k} \omega^{\prime}, X_{k+1} \omega^{\prime}\right)\right) \wedge \bigwedge_{k=0}^{n-1} \tilde{q}_{k}\left(X_{k} \omega, X_{k+1} \omega\right) \\
= & \sup _{\omega \in \Lambda: \omega(0)=x} h(\omega) \wedge q(\omega) \\
= & f_{\Lambda} h \mathrm{~d} P_{x} .
\end{aligned}
$$

Therefore $E\left(h \mid \mathcal{M}_{n}\right)$ satisfies the equation (1.3). Next let $\alpha \in[0,1]$. Noting

$$
h \in \mathcal{F}(\mathcal{M}) \text { and } \bigwedge_{k=0}^{n-1} 1_{E_{k} \times E_{k+1}}\left(X_{k}, X_{k+1}\right) \wedge \bigwedge_{k=n}^{\infty} \tilde{q}_{k}\left(X_{k}, X_{k+1}\right) \in \mathcal{F}(\mathcal{M}),
$$

we have $\Gamma:=\left\{\omega^{\prime} \in \Omega \mid h\left(\omega^{\prime}\right) \wedge \bigwedge_{k=n}^{\infty} \tilde{q}_{k}\left(X_{k} \omega^{\prime}, X_{k+1} \omega^{\prime}\right) \geq \alpha\right\} \in \mathcal{E}(\Omega)$. From Lemma 1.1(ii), we obtain

$$
\begin{aligned}
& \left\{\omega \in \Omega \mid E\left(h \mid \mathcal{M}_{n}\right)(\omega) \geq \alpha\right\} \\
& \quad=\bigcup_{\omega^{\prime} \in \Omega_{n+1}}\left\{\omega \in \Omega \mid h\left(\omega(0), \omega(1), \cdots, \omega(n), \omega^{\prime}(n+1), \cdots\right) \wedge q\left(\omega(n), \omega^{\prime}(n+1), \cdots\right) \geq \alpha\right\} \\
& \quad=\operatorname{proj}_{E_{0} \times E_{1} \times \cdots \times E_{n}}(\Gamma) \times \Omega_{n+1} \in \mathcal{E}(\Omega) \cap \mathcal{M}_{n} .
\end{aligned}
$$

By (1.4) and Lemma 1.1, we get $E\left(h \mid \mathcal{M}_{n}\right) \in \mathcal{F}\left(\mathcal{M}_{n}\right)$.

Finally we show the uniqueness of solutions of (1.3). Put $g=E\left(h \mid \mathcal{M}_{n}\right)$ and let another $\mathcal{M}_{n}$-measurable fuzzy set $g^{\prime}$ also satisfy (1.3). For $\epsilon>0$ and $x \in E$ we put

$\Lambda_{\epsilon, x}:=\left\{\omega \in \Omega \mid \omega(0)=x, g(\omega) \wedge \bigwedge_{k=0}^{n-1} \tilde{q}_{k}\left(X_{k} \omega, X_{k+1} \omega\right) \geq g^{\prime}(\omega) \wedge \bigwedge_{k=0}^{n-1} \tilde{q}_{k}\left(X_{k} \omega, X_{k+1} \omega\right)+\epsilon\right\} \in \mathcal{M}_{n}$. 
Let $\epsilon>0$ and $x \in E$. If $\Lambda_{\epsilon, x} \neq \emptyset$, then we have

$$
\begin{aligned}
f_{\Lambda_{\epsilon, x}} g^{\prime} \mathrm{d} P_{x} & =f_{\Lambda_{\epsilon, x}} h \mathrm{~d} P_{x}=f_{\Lambda_{\epsilon, x}} g \mathrm{~d} P_{x} \\
& =\sup _{\omega \in \Lambda_{\epsilon, x}}\left(g(\omega) \wedge \bigwedge_{k=0}^{n-1} \tilde{q}_{k}\left(X_{k} \omega, X_{k+1} \omega\right)\right) \\
& \geq \sup _{\omega \in \Lambda_{\epsilon, x}}\left(g^{\prime}(\omega) \wedge \bigwedge_{k=0}^{n-1} \tilde{q}_{k}\left(X_{k} \omega, X_{k+1} \omega\right)+\epsilon\right) \\
& =f_{\Lambda_{\epsilon, x}} g^{\prime} \mathrm{d} P_{x}+\epsilon .
\end{aligned}
$$

So we get $\Lambda_{\epsilon, x}=\emptyset$ for all $\epsilon>0$ and $x \in E$. Therefore

$$
g \wedge \bigwedge_{k=0}^{n-1} \tilde{q}_{k}\left(X_{k}, X_{k+1}\right) \leq g^{\prime} \wedge \bigwedge_{k=0}^{n-1} \tilde{q}_{k}\left(X_{k}, X_{k+1}\right) .
$$

Since the reverse inequality holds similarly; we obtain $g=g^{\prime}$ a.s..

Lemma 1.4. Let $h \in \mathcal{F}(\mathcal{M})$. Then :

(i) $E\left(E\left(h \mid \mathcal{M}_{m}\right) \mid \mathcal{M}_{n}\right)=E\left(h \mid \mathcal{M}_{n}\right)$ for $m, n \in \mathbf{N}(m \geq n)$;

(ii) $E\left(h \wedge 1_{\Lambda} \mid \mathcal{M}_{n}\right)=E\left(h \mid \mathcal{M}_{n}\right) \wedge 1_{\Lambda}$ a.s. for $n \in \mathbf{N}$ and $\Lambda \in \mathcal{E}(\Omega) \cap \mathcal{M}_{n}$;

(iii) $E\left(h \wedge g\left(X_{0}, X_{1}, \cdots, X_{n}\right) \mid \mathcal{M}_{n}\right)=E\left(h \mid \mathcal{M}_{n}\right) \wedge g\left(X_{0}, X_{1}, \cdots, X_{n}\right)$

for $n \in \mathbf{N}$ and $g \in \mathcal{F}\left(E_{0} \times E_{1} \times \cdots \times E_{n}\right)$;

(iv) $E\left(h \mid \mathcal{M}_{n}\right)=\bigvee_{m \in \bar{N}} E\left(h \wedge 1_{\Lambda_{m}} \mid \mathcal{M}_{n}\right)$

for $n \in \mathbf{N}$ and $\left\{\Lambda_{m}\right\}_{m=0}^{\infty} \subset \mathcal{E}(\Omega)$ such that $\left\{\Lambda_{m}\right\}_{m=0}^{\infty}$ is a partition of $\Omega$.

Proof. (i) Let $m, n \in \mathbf{N}(m \geq n)$. From the definition, we obtain

$$
\begin{aligned}
& E\left(E\left(h \mid \mathcal{M}_{m}\right) \mid \mathcal{M}_{n}\right)(\omega) \\
& =\sup _{\omega^{\prime} \in \Omega: \omega^{\prime}(k)=\omega(k)(k=0,1, \cdots, n)} E\left(h \mid \mathcal{M}_{m}\right)\left(\omega^{\prime}\right) \wedge \bigwedge_{k=n}^{\infty} \tilde{q}_{k}\left(X_{k} \omega^{\prime}, X_{k+1} \omega^{\prime}\right) \\
& =\sup _{\omega^{\prime} \in \Omega: \omega^{\prime}(k)=\omega(k)(k=0,1, \cdots, n)}\left(\sup _{\omega^{\prime \prime} \in \Omega: \omega^{\prime \prime}(k)=\omega^{\prime}(k)(k=0,1, \cdots, m)} h\left(\omega^{\prime \prime}\right) \wedge \bigwedge_{k=m}^{\infty} \tilde{q}_{k}\left(X_{k} \omega^{\prime \prime}, X_{k+1} \omega^{\prime \prime}\right)\right) \wedge \\
& =\sup _{\omega^{\prime} \in \Omega: \omega^{\prime}(k)=\omega(k)(k=0,1, \cdots, n)}^{m-1} \bigwedge_{k=n}^{m} \tilde{q}_{k}\left(X_{k} \omega^{\prime}, X_{k+1} \omega^{\prime}\right) \\
& =E\left(h \mid \mathcal{M}_{n}\right)(\omega) \text { for } \omega \in \Omega .
\end{aligned}
$$

(ii) Let $n \in \mathbf{N}$ and $\Lambda \in \mathcal{E}(\Omega) \cap \mathcal{M}_{n}$. From Proposition 1.2 , for $\Gamma \in \mathcal{M}_{n}$ and $x \in E$, we have

$$
f_{\Gamma} E\left(h \wedge 1_{\Lambda} \mid \mathcal{M}_{n}\right) \mathrm{d} P_{x}=f_{\Gamma} h \wedge 1_{\Lambda} \mathrm{d} P_{x}=f_{\Gamma \cap \Lambda} E\left(h \mid \mathcal{M}_{n}\right) \mathrm{d} P_{x}=f_{\Gamma} E\left(h \mid \mathcal{M}_{n}\right) \wedge 1_{\Lambda} \mathrm{d} P_{x}
$$


Therefore we obtain (ii).

(iii) Let $n \in \mathbf{N}$ and $g \in \mathcal{F}\left(E_{0} \times E_{1} \times \cdots \times E_{n}\right)$. Then

$$
\begin{aligned}
E & \left(h \wedge g\left(X_{0}, X_{1}, \cdots, X_{n}\right) \mid \mathcal{M}_{n}\right)(\omega) \\
= & \sup _{\omega^{\prime} \in \Omega: \omega^{\prime}(k)=\omega(k)(k=0,1, \cdots, n)} h\left(\omega^{\prime}\right) \wedge g\left(\omega^{\prime}(0), \omega^{\prime}(1), \cdots, \omega^{\prime}(n)\right) \wedge \bigwedge_{k=n}^{\infty} \tilde{q}_{k}\left(X_{k} \omega^{\prime}, X_{k+1} \omega^{\prime}\right) \\
& =\sup _{\omega^{\prime} \in \Omega: \omega^{\prime}(k)=\omega(k)(k=0,1, \cdots, n)} h\left(\omega^{\prime}\right) \wedge \bigwedge_{k=n}^{\infty} \tilde{q}_{k}\left(X_{k} \omega^{\prime}, X_{k+1} \omega^{\prime}\right) \wedge g(\omega(0), \omega(1), \cdots, \omega(n)) \\
& =E\left(h \mid \mathcal{M}_{n}\right)(\omega) \wedge g\left(X_{0}, X_{1}, \cdots, X_{n}\right)(\omega) \text { for } \omega \in \Omega .
\end{aligned}
$$

(iv) can be easily checked from the definition.

Next we consider stopping times.

Definition. A map $\tau: \Omega \mapsto \overline{\mathbf{N}}$ is called a $\left(\left\{\mathcal{M}_{n}\right\}_{n \in \mathrm{N}^{-}}\right.$adapted $)$stopping time if

$$
\{\tau=n\} \in \mathcal{M}_{n} \quad \text { for all } n \in \mathbf{N} .
$$

And we call a map $\tau: \Omega \mapsto \overline{\mathbf{N}}$ an $\mathcal{E}$-stopping time if

$$
\{\tau=n\} \in \mathcal{M}_{n} \cap \mathcal{E}(\Omega) \text { for all } n \in \mathbf{N} \text {. }
$$

It is trivial that a constant stopping time, i.e. $\tau=n_{0}$ for some $n_{0} \in \mathbf{N}$, is an $\mathcal{E}$-stopping time. Further the following lemmas hold.

Definition ([11]). For a set $A \in \mathcal{B}(E)$, we define a first entry time, $\tau_{A}$, and a first hitting time, $\sigma_{A}$, of $A$ by

$$
\begin{gathered}
\tau_{A}(\omega)=\inf \left\{n \mid n \in \mathbf{N}, X_{n}(\omega) \in A\right\} \quad \text { for } \omega \in \Omega ; \\
\sigma_{A}(\omega)=\inf \left\{n \geq 1 \mid n \in \mathbf{N}, X_{n}(\omega) \in A\right\} \quad \text { for } \omega \in \Omega,
\end{gathered}
$$

where the infima of the empty set are understood to be $+\infty$.

Lemma 1.5. For a set $A$ such that $A \in \mathcal{E}(E)$ and $A^{c} \in \mathcal{E}(E), \tau_{A}$ and $\sigma_{A}$ are $\mathcal{E}$-stopping times.

Proof. From Lemma 1.1(i), we have

$$
\left\{\tau_{A}=n\right\}=\bigcap_{k=0}^{n-1}\left\{X_{k} \in A^{c}\right\} \cap\left\{X_{n} \in A\right\} \in \mathcal{M}_{n} \cap \mathcal{E}(\Omega) \text { for all } n \in \mathbf{N} .
$$

Therefore $\tau_{A}$ is an $\mathcal{E}$-stopping time. We can check $\sigma_{A}$ similarly.

Lemma 1.6. Let $\sigma$ and $\tau$ be $\mathcal{E}$-stopping times. Then $\sigma \vee \tau, \sigma \wedge \tau$ and $\sigma+\tau \circ \theta_{\sigma}$ are $\mathcal{E}$-stopping times. 
Proof. Let $\sigma$ and $\tau$ be $\mathcal{E}$-stopping times. Then we obtain that $\sigma \vee \tau$ and $\sigma \wedge \tau$ are $\mathcal{E}$-stopping times from Lemma 1.1(i) and the following facts : For all $n \in \mathbf{N}$,

$$
\begin{gathered}
\{\sigma \wedge \tau=n\}=\bigcup_{k=0}^{n}(\{\sigma=n\} \cap\{\tau=k\}) \cup \bigcup_{k=0}^{n-1}(\{\sigma=k\} \cap\{\tau=n\}) \in \mathcal{E}(\Omega) \cap \mathcal{M}_{n} \\
\{\sigma \vee \tau=n\}=\bigcup_{k=n}^{\infty}(\{\sigma=n\} \cap\{\tau=k\}) \cup \bigcup_{k=n+1}^{\infty}(\{\sigma=k\} \cap\{\tau=n\}) \in \mathcal{E}(\Omega) \cap \mathcal{M}_{n} .
\end{gathered}
$$

Further since $\{\tau=n-k\} \in \mathcal{E}(\Omega) \cap \mathcal{M}_{n-k}(k=0,1,2 \cdots, n), \theta_{k}^{-1}\{\tau=n-k\}=$ $\{\omega \mid \tau(\omega(k+1), \omega(k+2), \cdots)=n-k\} \in \mathcal{E}(\Omega) \cap \mathcal{M}_{n}$. Therefore

$$
\left\{\sigma+\tau \circ \theta_{\sigma}=n\right\}=\bigcup_{k=0}^{n}\left(\{\sigma=k\} \cap \theta_{k}^{-1}\{\tau=n-k\}\right) \in \mathcal{E}(\Omega) \cap \mathcal{M}_{n} .
$$

So $\sigma+\tau \circ \theta_{\sigma}$ is an $\mathcal{E}$-stopping time.

Definition ([7]). Let $\tau$ be a stopping time. We define a sub- $\sigma$-field $\mathcal{M}_{\tau}$ of $\mathcal{M}$ by

$$
\mathcal{M}_{\tau}:=\left\{\Lambda \in \mathcal{M} \mid \Lambda \cap\{\tau=n\} \in \mathcal{M}_{n} \text { for all } n \in \mathbf{N}\right\} .
$$

For an $\mathcal{E}$-stopping time $\tau$, we can define a fuzzy expectation with $\mathcal{M}_{\tau}$.

Proposition 1.3. For $h \in \mathcal{F}(\mathcal{M})$ and an $\mathcal{E}$-stopping time $\tau$, we define

$$
E\left(h \mid \mathcal{M}_{\tau}\right)=E\left(h \mid \mathcal{M}_{n}\right) \quad \text { on }\{\tau=n\} \quad \text { for } n \in \overline{\mathbf{N}}
$$

Then $E\left(h \mid \mathcal{M}_{\tau}\right)$ is a fuzzy set in $\mathcal{F}\left(\mathcal{M}_{\tau}\right)$ and is the unique element in $\mathcal{F}\left(\mathcal{M}_{\tau}\right)$ (in the sense of a.s. ) such that

$$
f_{\Lambda} E\left(h \mid \mathcal{M}_{\tau}\right) d P_{x}=f_{\Lambda} h d P_{x} \quad \text { for all } \Lambda \in \mathcal{M}_{\tau} \text { and } x \in E
$$

Proof. Let $h \in \mathcal{F}(\Omega)$ and let $\tau$ be an $\mathcal{E}$-stopping time. Let $\Lambda \in \mathcal{M}_{\tau}$ and put $\Lambda_{n}:=$ $\Lambda \cap\{\tau=n\}$ for $n \in \overline{\mathbf{N}}$. From Lemma 1.2(ii) and Proposition 1.2, for any $x \in E$

$$
f_{\Lambda} E\left(h \mid \mathcal{M}_{\tau}\right) \mathrm{d} P_{x}=\bigvee_{n \in \overline{\mathrm{N}}} f_{\Lambda_{n}} E\left(h \mid \mathcal{M}_{\tau}\right) \mathrm{d} P_{x}=\bigvee_{n \in \overline{\mathrm{N}}} f_{\Lambda_{n}} E\left(h \mid \mathcal{M}_{n}\right) \mathrm{d} P_{x}=f_{\Lambda} h \mathrm{~d} P_{x} .
$$

Thus $E\left(h \mid \mathcal{M}_{\tau}\right)$ satisfies (1.5). Further, using Lemma 1.1(i) and Proposition 1.2, for all $n \in \overline{\mathbf{N}}$ and $\alpha \in[0,1]$

$$
\left\{E\left(h \mid \mathcal{M}_{\tau}\right) \geq \alpha\right\} \cap\{\tau=n\}=\left\{E\left(h \mid \mathcal{M}_{n}\right) \geq \alpha\right\} \cap\{\tau=n\} \in \mathcal{E}(\Omega) \cap \mathcal{M}_{n} .
$$

Therefore we obtain $\left\{E\left(h \mid \mathcal{M}_{\tau}\right) \geq \alpha\right\} \in \mathcal{E}(\Omega) \cap \mathcal{M}_{\tau}$ for all $\alpha \in[0,1]$. The other property can be checked in the same line as Proposition 1.2.

Corollary 1.1. Let $\left\{Y_{n}\right\}_{n \in \overline{\mathbf{N}}}$ be a sequence satisfying $Y_{n} \in \mathcal{F}\left(M_{n}\right)(n \in \overline{\mathbf{N}})$ and let $\tau$ be an $\mathcal{E}$-stopping time. Define

$$
Y_{\tau}=Y_{n} \quad \text { on }\{\tau=n\} \quad \text { for } n \in \overline{\mathbf{N}} .
$$

Then $Y_{\tau} \in \mathcal{F}\left(\mathcal{M}_{\tau}\right)$. 


\section{Markov property for the fuzzy process}

In this section we show a Markov property for the fuzzy process $\left\{X_{n}\right\}_{n \in \mathrm{N}}$ with the possibility measure $P$.

Definition. Let $\tau$ be an $\mathcal{E}$-stopping time. We define

$$
X_{\tau}=X_{n} \quad \text { on }\{\tau=n\} \quad \text { for } n \in \overline{\mathbf{N}} \text {. }
$$

Lemma 2.1. Let $\tilde{s} \in \mathcal{F}(E)$. Then :

(i) $\tilde{s}\left(X_{n}\right) \in \mathcal{F}\left(\mathcal{M}_{n}\right)$ for $n \in \mathbf{N}$;

(ii) $\tilde{s}\left(X_{\tau}\right) \in \mathcal{F}\left(\mathcal{M}_{\tau}\right)$ for an $\mathcal{E}$-stopping time $\tau$.

Proof. (i) Let $\tilde{s} \in \mathcal{F}(E)$ and $n \in \mathbf{N}$. Since $\{x \in E \mid \tilde{s}(x) \geq \alpha\} \in \mathcal{E}(E)$, for $\alpha \in[0,1]$

$$
\left\{\tilde{s}\left(X_{n}\right) \geq \alpha\right\}=\{\omega \mid \tilde{s}(\omega(n)) \geq \alpha\}=\prod_{k=0}^{n-1} E_{k} \times\{x \in E \mid \tilde{s}(x) \geq \alpha\} \times \prod_{k=n+1}^{\infty} E_{k} \in \mathcal{E}(\Omega) \cap \mathcal{M}_{n}
$$

Therefore $\tilde{s}\left(X_{n}\right) \in \mathcal{F}\left(\mathcal{M}_{n}\right)$. (ii) Let $\tilde{s} \in \mathcal{F}(E)$ and let $\tau$ be an $\mathcal{E}$-stopping time. From (i) and Corollary 1.1, we obtain $\tilde{s}\left(X_{\tau}\right) \in \mathcal{F}\left(\mathcal{M}_{\tau}\right)$.

Owing to Lemmas 1.2(i) and 2.1, we may introduce the following fuzzy transitions.

Definition. For $n \in \mathbf{N}$ and an $\mathcal{E}$-stopping time $\tau$, we define maps, $P_{n}$ and $P_{\tau}, \mathcal{F}(E) \mapsto$ $\mathcal{F}(E)$ by

$$
\begin{array}{ll}
P_{n} \tilde{s}(x):=E_{x}\left(\tilde{s}\left(X_{n}\right)\right)(x \in E) & \text { for } \tilde{s} \in \mathcal{F}(E) \\
P_{\tau} \tilde{s}(x):=E_{x}\left(\tilde{s}\left(X_{\tau}\right)\right)(x \in E) & \text { for } \tilde{s} \in \mathcal{F}(E) .
\end{array}
$$

We call $P_{n}$ and $P_{\tau}$ fuzzy transitions.

Regarding the fuzzy process $\left\{X_{n}\right\}_{n \in \mathrm{N}}$, we obtain a Markov property and a strong Markov property like the theory of Markov chains in Revuz [11].

Theorem 2.1. For $h \in \mathcal{F}(\mathcal{M})$, the following Markov property holds :

$$
E\left(h \circ \theta_{n} \mid \mathcal{M}_{n}\right)=E_{X_{n}}(h) \text { a.s. for } n \in \mathbf{N}
$$

Especially for $\tilde{s} \in \mathcal{F}(E)$,

$$
E\left(\tilde{s}\left(X_{m+n}\right) \mid \mathcal{M}_{n}\right)=P_{m} \tilde{s}\left(X_{n}\right) \text { a.s. } \quad \text { for } m, n \in \mathbf{N} \text {. }
$$


Proof. Let $h \in \mathcal{F}(\mathcal{M})$. Then for all $\Lambda \in \mathcal{M}_{n}$ and all $x \in E$ we have

$$
\begin{aligned}
f_{\Lambda} E\left(h \circ \theta_{n} \mid \mathcal{M}_{n}\right) \mathrm{d} P_{x} & =f_{\Lambda} h \circ \theta_{n} \mathrm{~d} P_{x} \\
& =\sup _{\omega \in \Lambda: \omega(0)=x} h\left(\theta_{n} \omega\right) \wedge \bigwedge_{k=0}^{\infty} \tilde{q}_{k}\left(X_{k} \omega, X_{k+1} \omega\right) \\
& =\sup _{\omega \in \Lambda: \omega(0)=x}\left(\sup _{\omega^{\prime} \in \Omega: \omega^{\prime}(0)=\omega(n)} h\left(\omega^{\prime}\right) \wedge q\left(\omega^{\prime}\right)\right) \wedge \bigwedge_{k=0}^{n} \tilde{q}_{k}\left(X_{k} \omega, X_{k+1} \omega\right) \\
& =f_{\Lambda} E_{X_{n}}(h) \mathrm{d} P_{x} .
\end{aligned}
$$

Thus we obtain (2.1) in the same arguments as the proof of the uniqueness in Proposition 1.2. Further, for $\tilde{s} \in \mathcal{F}(E)$, we have

$E\left(\tilde{s}\left(X_{m+n}\right) \mid \mathcal{M}_{n}\right)=E\left(\tilde{s}\left(X_{m} \circ \theta_{n}\right) \mid \mathcal{M}_{n}\right)=E_{X_{n}}\left(\tilde{s}\left(X_{m}\right)\right)=P_{m} \tilde{s}\left(X_{n}\right)$ a.s. for $m, n \in \mathbf{N}$.

Therefore the proof is completed.

Theorem 2.2. For $h \in \mathcal{F}(\mathcal{M})$, the following strong Markov property holds :

$$
E\left(h \circ \theta_{\tau} \mid \mathcal{M}_{\tau}\right)=E_{X_{\tau}}(h) \text { a.s. } \quad \text { for a finite } \mathcal{E}_{\text {-stopping time } \tau}
$$

Further for $\tilde{s} \in \mathcal{F}(E)$,

$$
E\left(\tilde{s}\left(X_{\sigma+\tau \circ \theta_{\sigma}}\right) \mid \mathcal{M}_{\sigma}\right)=P_{\tau} \tilde{s}\left(X_{\sigma}\right) \text { a.s. for finite } \mathcal{E} \text {-stopping times } \sigma \text { and } \tau \text {. }
$$

Proof. Let $h \in \mathcal{F}(\mathcal{M})$ and $\tau$ be a finite $\mathcal{E}$-stopping time. Let $\Lambda \in \mathcal{M}_{\tau}$ and put $\Lambda_{n}:=\Lambda \cap\{\tau=n\}$ for $n \in \overline{\mathbf{N}}$. Then, using Markov property and Lemma 1.2(ii), for all $x \in E$ we have

$$
\begin{aligned}
f_{\Lambda} E\left(h \circ \theta_{\tau} \mid \mathcal{M}_{\tau}\right) \mathrm{d} P_{x} & =\bigvee_{n \in \mathrm{N}} f_{\Lambda_{n}} E\left(h \circ \theta_{\tau} \mid \mathcal{M}_{n}\right) \mathrm{d} P_{x} \\
& =\bigvee_{n \in \mathrm{N}} f_{\Lambda_{n}} h \circ \theta_{\tau} \mathrm{d} P_{x} \\
& =\bigvee_{n \in \mathrm{N}} f_{\Lambda_{n}} h \circ \theta_{n} \mathrm{~d} P_{x} \\
& =\bigvee_{n \in \mathrm{N}} f_{\Lambda_{n}} E_{X_{n}}(h) \mathrm{d} P_{x} \\
& =f_{\Lambda} E_{X_{\tau}}(h) \mathrm{d} P_{x}
\end{aligned}
$$

So we obtain (2.3). Since (2.4) is trivial from (2.3), we obtain this theorem.

Corollary 2.1. It holds that

$$
P_{\sigma} P_{\tau}=P_{\sigma+\tau \circ \theta_{\sigma}} \text { on } \mathcal{F}(E) \text { for finite } \mathcal{E} \text {-stopping times } \sigma \text { and } \tau \text {. }
$$


From now on, we call $\left\{X_{n}\right\}_{n \in \mathrm{N}}$ a Markov fuzzy process, because of Theorems 2.1 and 2.2. For Sections 3 and 4 , we extend the state space $E$, referring the theory of Markov chains in Revuz [11, p.23]. Let $\Delta$ be not a point of $E$ and put $E_{\Delta}:=E \cup\{\Delta\}$ and $\Omega_{\Delta}:=\prod_{k=0}^{\infty} E_{\Delta}$. Since $\{E\} \in \mathcal{E}\left(E_{\Delta}\right)$ and $\{\Delta\} \in \mathcal{E}\left(E_{\Delta}\right)$, we can consistently extend the state space $E$ to $E_{\Delta}$ and the path space $\Omega$ to $\Omega_{\Delta}$. We also extend Markov fuzzy process $\left\{X_{n}\right\}_{n \in \mathrm{N}}$ and the shift $\left\{\theta_{n}\right\}_{n \in \mathrm{N}}$ :

$$
\begin{gathered}
X_{\tau}:= \begin{cases}X_{n} & \text { on }\{\tau=n\} \quad \text { for } n \in \mathbf{N} \\
\Delta & \text { on }\{\tau=\infty\} ;\end{cases} \\
\theta_{n}(\omega):= \begin{cases}\theta_{n}(\omega) \text { for } n \in \mathbf{N} \text { and } \omega \in \Omega_{\Delta} \\
\omega_{\Delta} & \text { for } n=\infty \text { and } \omega \in \Omega_{\Delta},\end{cases}
\end{gathered}
$$

where $\omega_{\Delta}=(\Delta, \Delta, \Delta, \cdots) \in \Omega_{\Delta}$. We let $\zeta$ be an entry time to $\{\Delta\}$, which is called the death time:

$$
\zeta(\omega):=\inf \left\{n \mid n \in \mathbf{N}, X_{n}(\omega)=\Delta\right\} \quad \text { for } \omega \in \Omega_{\Delta} .
$$

Then $\zeta$ is an $\mathcal{E}$-stopping time. A fuzzy set $\tilde{s} \in \mathcal{F}(E)$ is also extended to a fuzzy set on $E_{\Delta}$ :

$$
\tilde{s}(x):= \begin{cases}\tilde{s}(x) & \text { if } x \in E \\ 0 & \text { if } x=\Delta .\end{cases}
$$

Then the possibility measure $P_{x}, x \in E$, is extended to $P_{x}, x \in E_{\Delta}$, by

$$
P_{\Delta}(\Lambda):=\left\{\begin{array}{ll}
1 & \text { if } \omega_{\Delta} \in \Lambda \\
0 & \text { otherwise }
\end{array} \quad \text { for } \Lambda \in \sigma\left(X_{0}, X_{1}, X_{2}, \cdots\right) .\right.
$$

Since $E_{\Delta}$ and $\Omega_{\Delta}$ are compact by Hausdorff's one-point compactification, the same results hold for the extended Markov fuzzy processes without assuming (E.i) in the definition of $\mathcal{E}(S)$. From now on, this paper deals with the Markov fuzzy processes on the extended state space $E_{\Delta}$.

\section{An extension of the fuzzy expectations}

Let $S$ be a metric space. For fuzzy sets $\tilde{s}$ and $\tilde{r}$ on $S$, a relation $\tilde{s} \geq \tilde{r}$ means that $\tilde{s}(x) \geq \tilde{r}(x)$ for all $x \in S$. Then $\geq$ becomes a partial order. The possibility measure $P$ has the following properties.

Lemma 3.1. Let $\left\{h_{m}\right\}_{m=0}^{\infty} \subset \mathcal{F}(\mathcal{M})$. Then :

(i) $\bigvee_{m=0}^{\infty} E_{x}\left(h_{m}\right)=E_{x}\left(\bigvee_{m=0}^{\infty} h_{m}\right)$ for $x \in E$;

(ii) If $\left\{h_{m}\right\}_{m=0}^{\infty}$ is non-decreasing (with respect to the order $\geq$ ), then $\lim _{m \rightarrow \infty} E_{x}\left(h_{m}\right)=E_{x}\left(\lim _{m \rightarrow \infty} h_{m}\right)$ for $x \in E ;$

(iii) $\bigvee_{m=0}^{\infty} E\left(h_{m} \mid \mathcal{M}_{n}\right)=E\left(\bigvee_{m=0}^{\infty} h_{m} \mid \mathcal{M}_{n}\right)$ for $n \in \mathbf{N}$; 
(iv) If $\left\{h_{m}\right\}_{m=0}^{\infty}$ is non-decreasing (with respect to the order $\geq$ ), then $\lim _{m \rightarrow \infty} E\left(h_{m} \mid \mathcal{M}_{n}\right)=E\left(\lim _{m \rightarrow \infty} h_{m} \mid \mathcal{M}_{n}\right)$ for $n \in \mathbf{N}$.

Proof. (i) For $x \in E$,

$$
\bigvee_{m=0}^{\infty} E_{x}\left(h_{m}\right)=\bigvee_{m=0}^{\infty} \sup _{\omega \in \Omega: \omega(0)=x} h_{m}(\omega) \wedge q(\omega)=\sup _{\omega \in \Omega: \omega(0)=x} \bigvee_{m=0}^{\infty} h_{m}(\omega) \wedge q(\omega)=E_{x}\left(\bigvee_{m=0}^{\infty} h_{m}\right)
$$

So we get (i). The other equations are similar.

Let $S$ be a metric space such as $S=E$ or $S=\Omega$. In order to solve Snell's optimal stopping problem in the next section, $\mathcal{F}(S)$ is not a sufficient class of fuzzy sets. We extend $\mathcal{F}(S)$ and we define fuzzy expectations for them. Lemma 3.1 guarantees an extension of the expectations. Let

$$
\begin{aligned}
\mathcal{G}(S):=\{\text { fuzzy sets } \tilde{s} \mid & \text { there exists a sequence }\left\{\tilde{s}_{n}\right\}_{n=0}^{\infty} \subset \mathcal{F}(S) \\
& \text { such that } \left.\bigvee_{n=0}^{\infty} \tilde{s}_{n}=\tilde{s}\right\} .
\end{aligned}
$$

Then we can take $\left\{\tilde{s}_{n}\right\}_{n=0}^{\infty} \subset \mathcal{F}(S)$ to be non-decreasing, considering $\tilde{s}_{n}^{\prime}:=\bigvee_{k \leq n} \tilde{s}_{k} \in$ $\mathcal{F}(S)(n=0,1,2, \cdots)$. Further $h(\in \mathcal{G}(S))$ is $\mathcal{B}(S)$-measurable and $\mathcal{F}^{0}(S) \subset \mathcal{F}(S) \subset \mathcal{G}(S)$. Using Lemma 3.1(ii)(iv), we define fuzzy expectations for $h \in \mathcal{G}(\mathcal{M})$ :

For $h \in \mathcal{G}(\mathcal{M})$ there exists a non-decreasing sequence $\left\{h_{n}\right\}_{n \in \mathrm{N}} \subset \mathcal{F}(\mathcal{M})$ such that $\lim _{n \rightarrow \infty} h_{n}=\tilde{s}$. Then we define

$$
\begin{aligned}
E_{x}(h) & :=\lim _{n \rightarrow \infty} E_{x}\left(h_{n}\right) \quad \text { for } x \in E \\
E\left(h \mid \mathcal{M}_{n}\right) & =\lim _{m \rightarrow \infty} E\left(h_{m} \mid \mathcal{M}_{n}\right) \quad \text { for } n \in \mathbf{N} .
\end{aligned}
$$

(3.1) and (3.2) are well-defined since $E_{x}(\cdot)$ keeps the monotonicity for fuzzy sets (see Lemma 3.1(i)) and $\mathcal{F}(\mathcal{M})$ is directed upwards, i.e. $h \vee g \in \mathcal{F}(\mathcal{M})$ for $h, g \in \mathcal{F}(\mathcal{M})$. Then we have $E .(h) \in \mathcal{G}(E)$ and $E\left(h \mid \mathcal{M}_{\tau}\right) \in \mathcal{G}\left(\mathcal{M}_{\tau}\right)$ for an $\mathcal{E}$-stopping time $\tau$. Conversely for $\tilde{s}(\in \mathcal{G}(E))$, we can easily check $\tilde{s}\left(X_{\tau}\right) \in \mathcal{G}\left(\mathcal{M}_{\tau}\right)$ for an $\mathcal{E}$-stopping time $\tau$. Therefore, from now on, we can use the results in Sections $1-3$, replacing $\mathcal{F}(E)$ and $\mathcal{F}(\Omega)$ with the extended $\mathcal{G}(S)$ and $\mathcal{G}(\Omega)$ respectively.

\section{Snell's problem for Markov fuzzy processes}

As an application of Markov fuzzy processes, we consider Snell's problem (c.f. Neveu [7, Sect.VI-2]) :

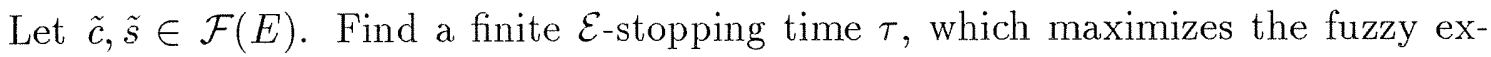
pected value of fuzzy goals $E_{x}\left(\bigwedge_{k=0}^{\tau-1} \tilde{c}\left(X_{k}\right) \wedge \tilde{s}\left(X_{\tau}\right)\right)(x \in E)$. 
We put an optimal value

$$
\tilde{v}(x):=\sup _{\tau: \mathcal{E} \text {-stopping times }} E_{x}\left(\bigwedge_{k=0}^{\tau-1} \tilde{c}\left(X_{k}\right) \wedge \tilde{s}\left(X_{\tau}\right)\right), \quad x \in E .
$$

Define a fuzzy transition $Q$ by $Q \tilde{r}:=\tilde{c} \wedge P \tilde{r}$ for $\tilde{r} \in \mathcal{G}(E)$. Further we put fuzzy transitions $Q_{0}:=I$ (identity map) and $Q_{n+1}:=Q Q_{n}$ for $n \in \mathbf{N}$. We introduce $Q$-superharmonic property for fuzzy sets.

Definition ([11]). A fuzzy set $\tilde{s}$ on $E$ is called $Q$-superharmonic if $\tilde{s} \in \mathcal{G}(E)$ and

$$
\tilde{s}(x) \geq Q \tilde{s}(x) \text { for all } x \in E .
$$

Lemma 4.1. Then we have the following (i) and (ii) :

(i) For $n \in \mathbf{N}$ it holds that

$$
Q_{n} \tilde{s}(x):=E_{x}\left(\bigwedge_{k=0}^{n-1} \tilde{c}\left(X_{k}\right) \wedge \tilde{s}\left(X_{n}\right)\right), \quad x \in E .
$$

(ii) We define

$$
\tilde{u}(x):=\bigvee_{n \in \mathrm{N}} Q_{n} \tilde{s}(x)=E_{x}\left(\bigvee_{n \in \mathbb{N}}\left(\bigwedge_{k=0}^{n-1} \tilde{c}\left(X_{k}\right) \wedge \tilde{s}\left(X_{n}\right)\right)\right), \quad x \in E .
$$

Then $\tilde{u}(\in \mathcal{G}(E))$ is the smallest $Q$-superharmonic fuzzy set dominating $\tilde{s}$ and satisfies

$$
\tilde{u}=\tilde{s} \vee Q \tilde{u}
$$

Proof. (i) We can easily check (4.1) by induction on $n$ from the Markov property and Lemma 1.4(iii). (ii) From the Markov property and Lemma 1.4(iii), for $x \in E$, we have

$$
\begin{aligned}
Q \tilde{u}(x) & =\tilde{c}(x) \wedge E_{x}\left(E_{X_{1}}\left(\bigvee_{n \in \mathrm{N}}\left(\bigwedge_{k=0}^{n-1} \tilde{c}\left(X_{k}\right) \wedge \tilde{s}\left(X_{n}\right)\right)\right)\right) \\
& =E_{x}\left(\tilde{c}\left(X_{0}\right) \wedge\left(\bigvee_{n \in \mathrm{N}}\left(\bigwedge_{k=0}^{n-1} \tilde{c}\left(X_{k}\right) \wedge \tilde{s}\left(X_{n}\right)\right)\right) \circ \theta_{1}\right) \\
& =E_{x}\left(\tilde{c}\left(X_{0}\right) \wedge \bigvee_{n \geq 1}\left(\bigwedge_{k=1}^{n-1} \tilde{c}\left(X_{k}\right) \wedge \tilde{s}\left(X_{n}\right)\right)\right) \\
& =E_{x}\left(\bigvee_{n \geq 1}\left(\bigwedge_{k=0}^{n-1} \tilde{c}\left(X_{k}\right) \wedge \tilde{s}\left(X_{n}\right)\right)\right) .
\end{aligned}
$$


Together with Lemma 3.1(i), for $x \in E$, we obtain

$$
\tilde{s}(x) \vee Q \tilde{u}(x)=E_{x}\left(\tilde{s}\left(X_{0}\right) \vee \bigvee_{n \geq 1}\left(\bigwedge_{k=0}^{n-1} \tilde{c}\left(X_{k}\right) \wedge \tilde{s}\left(X_{n}\right)\right)\right)=\tilde{u}(x) .
$$

Therefore $\tilde{u}$ satisfies (4.3) and $\tilde{u}$ is a $Q$-superharmonic fuzzy set dominating $\tilde{s}$. Further let $\tilde{g}$ be a $Q$-superharmonic fuzzy set dominating $\tilde{s}$. Then we have

$$
\tilde{g} \geq \bigvee_{n \in \mathrm{N}} Q_{n} \tilde{g} \geq \bigvee_{n \in \mathrm{N}} Q_{n} \tilde{s}=\tilde{u}
$$

Therefore we obtain this lemma since it is trivial that $\tilde{u} \in \mathcal{G}(E)$ from (4.2).

For an $\mathcal{E}$-stopping time $\tau$, by (4.1), we also define

$$
Q_{\tau} \tilde{s}(x):=E_{x}\left(\bigwedge_{k=0}^{\tau-1} \tilde{c}\left(X_{k}\right) \wedge \tilde{s}\left(X_{\tau}\right)\right), \quad x \in E
$$

Theorem 4.1. The optimal value is given by

$$
\tilde{v}=\tilde{u}
$$

Proof. Let $x \in E$. Since constant stopping times are $\mathcal{E}$-stopping times, for all finite $\mathcal{E}$-stopping times $\tau$, we have

$$
E_{x}\left(\bigwedge_{k=0}^{\tau-1} \tilde{c}\left(X_{k}\right) \wedge \tilde{s}\left(X_{\tau}\right)\right) \leq E_{x}\left(\bigvee_{n \in \mathrm{N}}\left(\bigwedge_{k=0}^{n-1} \tilde{c}\left(X_{k}\right) \wedge \tilde{s}\left(X_{n}\right)\right)\right)=\tilde{u}(x) .
$$

Therefore we obtain $\tilde{v}(x) \leq \tilde{u}(x)$. Reversely, from Lemmas 1.4(iii) and 3.1(i), we have

$$
\tilde{u}(x)=E_{x}\left(\bigvee_{n \in \mathbb{N}}\left(\bigwedge_{k=0}^{n-1} \tilde{c}\left(X_{k}\right) \wedge \tilde{s}\left(X_{n}\right)\right)\right)=\bigvee_{n \in \mathbb{N}} E_{x}\left(\bigwedge_{k=0}^{n-1} \tilde{c}\left(X_{k}\right) \wedge \tilde{s}\left(X_{n}\right)\right) \leq \tilde{v}(x)
$$

Therefore the proof is completed.

Lemma 4.2 (c.f. [7, Lem.VI-1-6]). It holds that

$$
\lim _{n \rightarrow \infty} Q_{n} \tilde{v}=\varlimsup_{n \rightarrow \infty} Q_{n} \tilde{s}
$$

Proof. Let $x \in E$. Using the Markov property and Lemma 3.1(i), for all $n \in \mathbf{N}$

$$
Q_{n} \tilde{u}(x)=E_{x}\left(\bigwedge_{k=0}^{n-1} \tilde{c}\left(X_{k}\right) \wedge \tilde{u}\left(X_{n}\right)\right)
$$




$$
\begin{aligned}
& =E_{x}\left(\bigwedge_{k=0}^{n-1} \tilde{c}\left(X_{k}\right) \wedge E_{X_{n}}\left(\bigvee_{m \in \mathrm{N}}\left(\bigwedge_{k=0}^{m-1} \tilde{c}\left(X_{k}\right) \wedge \tilde{s}\left(X_{m}\right)\right)\right)\right) \\
& =E_{x}\left(\bigvee_{m \geq n}\left(\bigwedge_{k=0}^{m-1} \tilde{c}\left(X_{k}\right) \wedge \tilde{s}\left(X_{m}\right)\right)\right) \\
& =\bigvee_{m \geq n} E_{x}\left(\bigwedge_{k=0}^{m-1} \tilde{c}\left(X_{k}\right) \wedge \tilde{s}\left(X_{m}\right)\right) .
\end{aligned}
$$

Since $\tilde{u}$ is $Q$-superharmonic from Lemma 4.1, the limit of the above equality exists when $n \rightarrow \infty$ and we obtain (4.5) together with Theorem 4.1 .

We give an optimal $\mathcal{E}$-stopping time under the following continuity condition and condition on $\tilde{s}$ for an initial state $x \in E$.

Condition (I). $\tilde{s}, \tilde{c}$ and $\tilde{q}_{k}(k=0,1,2, \cdots)$ are continuous.

Condition (II). It holds that $\overline{\lim }_{n \rightarrow \infty} Q_{n} \tilde{s}(x) \leq \tilde{s}(x)$.

Lemma 4.3. Under Condition (I),

$$
\tau_{0}:=\inf \left\{n \in \mathbf{N} \mid \tilde{v}\left(X_{n}\right)=\tilde{s}\left(X_{n}\right)\right\}
$$

is an $\mathcal{E}$-stopping time.

Proof. Suppose that Condition (I) holds. From Lemma 1.5, it is sufficient to check $\{\tilde{v}>\tilde{s}\} \in \mathcal{E}(E)$ and $\{\tilde{v}=\tilde{s}\} \in \mathcal{E}(E)$.

First we have $\{\tilde{s}<\alpha\}=\bigcup_{m=1}^{\infty}\{\tilde{s} \leq \alpha-1 / m\} \in \mathcal{E}(E)$ for $\alpha \in(0,1)$. On the other hand, from (4.4) and Lemma 1.1(i), we have $\{\tilde{v}>\alpha\}=\bigcup_{n \in \mathrm{N}} \bigcup_{m=1}^{\infty}\left\{Q_{n} \tilde{s} \geq \alpha+1 / m\right\} \in \mathcal{E}(E)$ for $\alpha \in(0,1)$. Therefore we obtain

$$
\{\tilde{v}>\tilde{s}\}=\bigcup_{\alpha \in \mathbf{Q} \cap(0,1)}(\{\tilde{v}>\alpha\} \cap\{\alpha>\tilde{s}\}) \in \mathcal{E}(E) \quad \text { for } \alpha \in(0,1),
$$

where $\mathbf{Q}$ denotes the set of all rational numbers.

Next, from (4.2) for $x \in E$ we have

$$
\begin{aligned}
\tilde{u}(x) & =E_{x}\left(\bigvee_{n \in N}\left(\bigwedge_{k=0}^{n-1} \tilde{c}\left(X_{k}\right) \wedge \tilde{s}\left(X_{n}\right)\right)\right) \\
& =\sup _{\omega \in \Omega: \omega(0)=x} \bigvee_{n \in N}\left(\bigwedge_{k=0}^{n-1} \tilde{c}(\omega(k)) \wedge \tilde{s}(\omega(n)) \wedge \bigwedge_{k=0}^{n-1} \tilde{q}_{k}(\omega(k), \omega(k+1))\right) .
\end{aligned}
$$

From Condition (I), $\tilde{u}$ is lower-semicontinuous. Therefore $\{\tilde{v}=\tilde{s}\}=\{\tilde{v} \leq \tilde{s}\}$ is closed. Thus $\tau_{0}$ is an $\mathcal{E}$-stopping time.

Theorem 4.2. Suppose that Condition (I) holds. Let $x \in E$. We also suppose that Condition (II) holds for $x$. Then $\tau_{0}$ is a finite optimal $\mathcal{E}$-stopping time for Snell's problem. 
In fact $\tau_{0}$ is bounded on the optimal paths in the sense that there exists $n_{0}\left(=n_{0}(x)\right) \in \mathbf{N}$, which depends only on $x$, such that

$$
\tilde{v}(x)=Q_{\tau_{0}} \tilde{s}(x)=Q_{\tau_{0} \wedge n} \tilde{s}(x) \quad \text { for all } n \geq n_{0} .
$$

Proof. We show this theorem, referring the proof of Neveu [7, Prop.VI-1-2]. Since we have $\tilde{u}\left(X_{n}\right)>\tilde{s}\left(X_{n}\right)$ on $\left\{n<\tau_{0}\right\}$, from (4.3) and the Markov property we obtain

$$
\tilde{u}\left(X_{n}\right)=Q \tilde{u}\left(X_{n}\right)=\tilde{c}\left(X_{n}\right) \wedge E\left(\tilde{u}\left(X_{n+1}\right) \mid \mathcal{M}_{n}\right) \text { a.s. for }\left\{n<\tau_{0}\right\} .
$$

Therefore, from Lemma 1.4(ii)-(iv), $\left\{\bigwedge_{k=0}^{\left(\tau_{0} \wedge n\right)-1} \tilde{c}\left(X_{k}\right) \wedge \tilde{u}\left(X_{\tau_{0} \wedge n}\right)\right\}_{n \in \mathrm{N}}$ has the following property like martingales in the probability theory :

$$
\begin{aligned}
& E\left(\bigwedge_{k=0}^{\left(\tau_{0} \wedge(n+1)\right)-1} \tilde{c}\left(X_{k}\right) \wedge \tilde{u}\left(X_{\tau_{0} \wedge(n+1)}\right) \mid \mathcal{M}_{n}\right) \\
& =E\left(\bigwedge_{k=0}^{\tau_{0}-1} \tilde{c}\left(X_{k}\right) \wedge \tilde{u}\left(X_{\tau_{0}}\right) \wedge 1_{\left\{\tau_{0} \leq n\right\}} \mid \mathcal{M}_{n}\right) \vee E\left(\bigwedge_{k=0}^{n} \tilde{c}\left(X_{k}\right) \wedge \tilde{u}\left(X_{n+1}\right) \wedge 1_{\left\{\tau_{0}>n\right\}} \mid \mathcal{M}_{n}\right) \\
& =\left(\bigwedge_{k=0}^{\tau_{0}-1} \tilde{c}\left(X_{k}\right) \wedge \tilde{u}\left(X_{\tau_{0}}\right) \wedge 1_{\left\{\tau_{0} \leq n\right\}}\right) \vee\left(\bigwedge_{k=0}^{n} \tilde{c}\left(X_{k}\right) \wedge E\left(\tilde{u}\left(X_{n+1}\right) \mid \mathcal{M}_{n}\right) \wedge 1_{\left\{\tau_{0}>n\right\}}\right) \\
& =\left(\bigwedge_{k=0}^{\tau_{0}-1} \tilde{c}\left(X_{k}\right) \wedge \tilde{u}\left(X_{\tau_{0}}\right) \wedge 1_{\left\{\tau_{0} \leq n\right\}}\right) \vee\left(\bigwedge_{k=0}^{n-1} \tilde{c}\left(X_{k}\right) \wedge \tilde{u}\left(X_{n}\right) \wedge 1_{\left\{\tau_{0}>n\right\}}\right) \\
& =\bigwedge_{k=0}^{\left(\tau_{0} \wedge n\right)-1} \tilde{c}\left(X_{k}\right) \wedge \tilde{u}\left(X_{\tau_{0} \wedge n}\right) \text { a.s.. }
\end{aligned}
$$

First we consider a case of $x \in\{\tilde{v}>\tilde{s}\}$. Together with Lemma 1.2 (ii), for $n \in \mathbf{N}$ we obtain

$$
\begin{aligned}
\tilde{u}(x) & =E_{x}\left(\bigwedge_{k=0}^{\left(\tau_{0} \wedge n\right)-1} \tilde{c}\left(X_{k}\right) \wedge \tilde{u}\left(X_{\tau_{0} \wedge n}\right)\right) \\
& =E_{x}\left(\bigwedge_{k=0}^{\tau_{0}-1} \tilde{c}\left(X_{k}\right) \wedge \tilde{u}\left(X_{\tau_{0}}\right) \wedge 1_{\left\{\tau_{0}<n\right\}}\right) \vee E_{x}\left(\bigwedge_{k=0}^{n-1} \tilde{c}\left(X_{k}\right) \wedge \tilde{u}\left(X_{n}\right) \wedge 1_{\left\{\tau_{0} \geq n\right\}}\right) .
\end{aligned}
$$

From Condition (II), we have $\varlimsup_{n \rightarrow \infty} Q_{n} \tilde{s}(x)<\tilde{v}(x)$ holds since $x \in\{\tilde{v}>\tilde{s}\}$. So there exists $n_{0}\left(=n_{0}(x)\right) \in \mathbf{N}$ such that

$$
\bigvee_{n \geq n_{0}} Q_{n} \tilde{s}(x)<\tilde{v}(x)
$$

From (4.9), we have

$E_{x}\left(\bigwedge_{k=0}^{n-1} \tilde{c}\left(X_{k}\right) \wedge \tilde{u}\left(X_{n}\right) \wedge 1_{\left\{\tau_{0} \geq n\right\}}\right) \leq Q_{n} \tilde{u}(x)=\bigvee_{m \geq n} Q_{m} \tilde{s}(x)<\tilde{v}(x)=\tilde{u}(x)$ for all $n \geq n_{0}$, 
(4.8) follows

$$
\tilde{u}(x)=E_{x}\left(\bigwedge_{k=0}^{\tau_{0}-1} \tilde{c}\left(X_{k}\right) \wedge \tilde{u}\left(X_{\tau_{0}}\right) \wedge 1_{\left\{\tau_{0}<n\right\}}\right) \text { for all } n \geq n_{0} .
$$

Together with Theorem 4.1 and the definition of $\tau_{0}$, we obtain

$$
\tilde{v}(x)=E_{x}\left(\bigwedge_{k=0}^{\tau_{0}-1} \tilde{c}\left(X_{k}\right) \wedge \tilde{s}\left(X_{\tau_{0}}\right) \wedge 1_{\left\{\tau_{0}<n\right\}}\right) \leq Q_{\tau_{0} \wedge n} \tilde{s}(x) \leq \tilde{v}(x) \text { for all } n \geq n_{0} .
$$

Therefore $\tilde{v}(x)=Q_{\tau_{0} \wedge n} \tilde{s}(x)$ for all $n \geq n_{0}$.

Further, noting

$$
\bigwedge_{k=0}^{\tau_{0}-1} \tilde{c}\left(X_{k}\right) \wedge \tilde{s}\left(X_{\tau_{0}}\right) \wedge 1_{\left\{\tau_{0}<n\right\}} \uparrow \bigwedge_{k=0}^{\tau_{0}-1} \tilde{c}\left(X_{k}\right) \wedge \tilde{s}\left(X_{\tau_{0}}\right) \wedge 1_{\left\{\tau_{0}<\infty\right\}} \quad \text { as } n \rightarrow \infty
$$

by Lemma 3.1(ii), (4.10) follows

$$
\begin{aligned}
\tilde{v}(x) & =\lim _{n \rightarrow \infty} E_{x}\left(\bigwedge_{k=0}^{\tau_{0}-1} \tilde{c}\left(X_{k}\right) \wedge \tilde{s}\left(X_{\tau_{0}}\right) \wedge 1_{\left\{\tau_{0}<n\right\}}\right) \\
& =E_{x}\left(\bigwedge_{k=0}^{\tau_{0}-1} \tilde{c}\left(X_{k}\right) \wedge \tilde{s}\left(X_{\tau_{0}}\right) \wedge 1_{\left\{\tau_{0}<\infty\right\}}\right) \\
& =Q_{\tau_{0}} \tilde{s}(x) .
\end{aligned}
$$

Consequently we get $\tilde{v}(x)=Q_{\tau_{0}} \tilde{s}(x)=Q_{\tau_{0} \wedge n} \tilde{s}(x)$ for $n \geq n_{0}$ in the case of $x \in\{\tilde{v}>\tilde{s}\}$. Since it is trivial that $\tilde{v}(x)=\tilde{s}(x)=Q_{0} \tilde{s}(x)$ if $x \in\{\tilde{v}=\tilde{s}\}$, we obtain (4.7).

Remark. We give an example satisfying Conditions (I) (II), using the dynamic fuzzy systems Yoshida et al. [14]. Let $d$ be a positive integer, let $\mathbf{R}^{d}$ be a $d$-dimensional Euclidean space and let $\mathbf{0}$ be its origin. Suppose that $\tilde{q}$ is linear, convex and contractive in the sense of [14, Assumption A and B]. Then we may consider that the state space is the positive orthant $\mathbf{R}_{+}^{d}:=\left\{\left(x^{1}, x^{2}, \cdots, x^{d}\right) \in \mathbf{R}^{d} \mid x^{i} \geq 0(i=1,2, \cdots, d)\right\}$ and that $\tilde{q}$ is continuous on $\mathbf{R}_{+}^{d} \times \mathbf{R}_{+}^{d} \backslash\{\mathbf{0}, \mathbf{0}\} \quad([14$, page 285$])$. $\mathbf{0}$ is a kind of irregular point since $\tilde{q}(\cdot, \mathbf{0})=1_{\{\mathbf{0}\}}$ from $[14,(2.4)]$. For simplicity, we suppose an absorbing assumption for $\mathbf{0}: \tilde{q}(\mathbf{0}, \cdot)=1_{\{\mathbf{0}\}}$ (see [14, Fig.2 of Sect.4]). Then 0 becomes an isolated absorbing point in the sense that

$$
X_{n} \begin{cases}=\mathbf{0}(n=1,2, \cdots) & P_{x^{-}} \text {a.s. } \text { if } x=\mathbf{0} \\ \in \mathbf{C}_{+}^{d}(n=1,2, \cdots) & P_{x^{-}} \text {-a.s. if } x \in \mathbf{C}_{+}^{d},\end{cases}
$$

where $\mathbf{C}_{+}^{d}:=\mathbf{R}_{+}^{d} \backslash\{\mathbf{0}\}$. Let $\tilde{c}$ and $\tilde{s}$ be continuous with $\tilde{s}(\mathbf{0})=1$. Then Condition (I) holds on the state space $\mathbf{C}_{+}^{d}$. Further Condition (II) also holds for all $x \in \mathbf{R}_{+}^{d}$ since, from (4.11) and $\left[14\right.$, Th.3.1], we have $P_{n} \tilde{s} \in \mathcal{F}^{0}\left(\mathbf{R}_{+}^{d}\right)$ and $\varlimsup_{n \rightarrow \infty} Q_{n} \tilde{s} \leq \varlimsup_{\lim _{n \rightarrow \infty}} P_{n} \tilde{s}=1_{\{0\}} \leq \tilde{s}$. A numerical example is given in [14, Sect.4]. 


\section{An application to fuzzy dynamic programming with stopping times}

Esogbue and Bellman [4, Sect.7] studied fuzzy dynamic programming under a transition possibility measure and they also studied fuzzy dynamic programming with a few kind of stopping times. We deal with fuzzy dynamic programming with finite stopping times depending on paths under a transition possibility measure.

We take state spaces $E$ and $E_{n}(n \in \mathbf{N})$ as before. Let an action space $U$ be also a finite-dimensional Euclidean space. Let $U_{0}, U_{1}, U_{2}, \cdots$ denote copies of $U$. Then the controlled path space is $\Omega:=\prod_{k=0}^{\infty}\left(E_{k} \times U_{k}\right)$. We also put $\Omega_{n}:=\prod_{k=n}^{\infty}\left(E_{k} \times U_{k}\right)$ for $n \in \mathbf{N}$. An element $\omega=(\omega(0), \pi(0), \omega(1), \pi(1), \omega(2), \pi(2), \cdots) \in \Omega$ is called a path controlled by a decision $\pi=(\pi(0), \pi(1), \pi(2), \cdots)$. We define maps $X_{n}(\omega):=\omega(n)$ and $\Pi_{n}(\omega):=\pi(n)$ for $\omega \in \Omega$ and $n \in \mathbf{N}$. We put $\sigma$-fields by $\mathcal{M}_{n}:=\sigma\left(X_{0}, \Pi_{0}, X_{1}, \Pi_{1}, \cdots, \Pi_{n-1}, X_{n}\right)$ for $n \in \mathrm{N}$ and $\mathcal{M}:=\sigma\left(\bigcup_{n \in \mathrm{N}} \mathcal{M}_{n}\right)$. Let $\tilde{q}_{n}:=\tilde{q}\left(\in \mathcal{F}\left(E_{n} \times U_{n} \times E_{n+1}\right)\right)$ be time-invariant continuous fuzzy relations $(n=0,1,2, \cdots)$ satisfying the following normality condition : For all $n \in \mathbf{N}$,

$$
\sup _{x \in E_{n}} \tilde{q}_{n}(x, u, y)=1\left((u, y) \in U_{n} \times E_{n+1}\right) \quad \text { and } \sup _{(u, y) \in U_{n} \times E_{n+1}} \tilde{q}_{n}(x, u, y)=1\left(x \in E_{n}\right) .
$$

It is easy to find such a fuzzy relation $\tilde{q}$ :

For example, take $E=U=\mathbf{R}^{1}$ and let $f: E \times U \mapsto E$ be a continuous function. Define

$$
\tilde{q}(x, u, y)=(1-|x-f(u, y)|) \vee 0, \quad(x, u, y) \in E \times U \times E .
$$

Then the fuzzy relation $\tilde{q}$ satisfies the conditions (c.f. [6, Fig.1],[14, Fig.2]).

We take $q:=\bigwedge_{n=0}^{\infty} \tilde{q}_{n}\left(X_{n}, \Pi_{n}, X_{n+1}\right) \in \mathcal{F}(\mathcal{M})$ and $P(\Lambda):=\sup _{\omega \in \Lambda} q(\omega)$ for $\Lambda \in \mathcal{M}$. For $h \in \mathcal{F}(\mathcal{M})$, we similarly define fuzzy expectations by the possibility measure $P$.

Now we consider the following fuzzy dynamic programming :

Let a fuzzy goal $\tilde{s}$ and a fuzzy constraint $\tilde{c}$ be continuous on $E$. Let a fuzzy constraint $\tilde{\mu}$ be continuous on $U$. Let $x \in E$. The problem is to find decisions $u_{0}, u_{1}, u_{2}, \cdots$, paths $x_{0}, x_{1}, x_{2}, \cdots$ and a finite optimal stopping time $\tau$ maximizing

$$
\bigwedge_{n=0}^{\tau-1}\left(\tilde{c}\left(x_{n}\right) \wedge \tilde{\mu}\left(u_{n}\right)\right) \wedge \tilde{s}\left(x_{\tau}\right)
$$

under the Markov fuzzy processes with the fuzzy transition :

$$
E_{x}(h)=\sup _{\omega: \omega(0)=x} h(\omega) \wedge \bigwedge_{n=0}^{\infty} \tilde{q}_{n}(\omega(n), \pi(n), \omega(n+1)) .
$$

Then, from (4.3) and (4.4), we obtain an optimality equation : For $n \in \mathbf{N}$,

$$
\tilde{v}\left(x_{n}\right)=\tilde{s}\left(x_{n}\right) \vee \sup _{\left(u_{n}, x_{n+1}\right) \in U_{n} \times E_{n+1}}\left\{\tilde{c}\left(x_{n}\right) \wedge \tilde{\mu}\left(u_{n}\right) \wedge \tilde{v}\left(x_{n+1}\right) \wedge \tilde{q}\left(x_{n}, u_{n}, x_{n+1}\right)\right\} .
$$


Especially taking $\tilde{c}=1_{E}$, it follows

$$
\tilde{v}\left(x_{n}\right)=\tilde{s}\left(x_{n}\right) \vee \sup _{\left(u_{n}, x_{n+1}\right) \in U_{n} \times E_{n+1}}\left\{\tilde{\mu}\left(u_{n}\right) \wedge \tilde{v}\left(x_{n+1}\right) \wedge \tilde{q}\left(x_{n}, u_{n}, x_{n+1}\right)\right\} .
$$

In $[4,(34)]$, they studied non-stopped fuzzy dynamic programming. We deal with the case with optimal stopping times when the state space $E$ and the action space $U$ are finite-dimensional Euclidean spaces. Under Condition (II), there exists $n_{0}\left(=n_{0}(x)\right) \in \mathbf{N}$ satisfying (4.9). From Theorem 4.2, we obtain the following fuzzy dynamic program :

$$
\begin{gathered}
\tilde{v}\left(x_{n}\right)=\tilde{s}\left(x_{n}\right) \vee \sup _{\left(u_{n}, x_{n+1}\right) \in U_{n} \times E_{n+1}}\left\{\tilde{\mu}\left(u_{n}\right) \wedge \tilde{v}\left(x_{n+1}\right) \wedge \tilde{q}\left(x_{n}, u_{n}, x_{n+1}\right)\right\} \quad \text { for } n<n_{0}, \\
\text { and } \tilde{v}\left(x_{n}\right)=\tilde{s}\left(x_{n_{0}}\right) \text { for } n \geq n_{0} .
\end{gathered}
$$

By solving the program backward, we can obtain an optimal path $\omega^{*}=\left(x, u_{0}^{*}, x_{1}^{*}, u_{1}^{*}, x_{2}^{*}, u_{2}^{*}, \cdots, x_{n_{0}}^{*}\right)$ an optimal decision $\pi^{*}=\left(u_{0}^{*}, u_{1}^{*}, u_{2}^{*}, \cdots, u_{n_{0}}^{*}\right)$, and an optimal stopping time $\tau_{0}\left(\omega^{*}\right)\left(\leq n_{0}\right)$.

\section{Acknowledgements}

The author would like to thank the referees for valuable comments and suggestions. The author also thanks to Prof. M. Kurano for his helpful advises.

\section{References}

[1] R.E.Bellman and L.A.Zadeh, Decision-making in a fuzzy environment, Management Sci. Ser B. 17 (1970) 141-164.

[2] D.P.Bertsekas and S.E.Shreve, Stochastic Optimal Control : the Discrete Time Case (Academic Press, New York, 1978).

[3] G.De Cooman and E.E.Kerre, Possibility theory: An integral theoretic approach, Fuzzy Sets and Systems 46 (1992) 287-299.

[4] A.O.Esogbue and R.E.Bellman, Fuzzy dynamic programming and its extensions, TIMS / Studies in Management Sci. 20 (North-Holland, Amsterdam, 1984) 147167.

[5] J.Kacprzyk, Decision making in a fuzzy environment with fuzzy termination time, Fuzzy Sets and Systems 1 (1978) 169-179.

[6] M.Kurano, M.Yasuda, J.Nakagami and Y.Yoshida, A limit theorem in some dynamic fuzzy systems, Fuzzy Sets and Systems 51 (1992) 83-88.

[7] J.Neveu, Discrete-Parameter Martingales (North-Holland, New York, 1975).

[8] K.P.Parthasarathy, Probability Measures on Metric Space (Academic Press, New York, 1967).

[9] M.L.Puri and D.Ralescu, A possibility measure is not a fuzzy measure, Fuzzy Sets and Systems 29 (1982) 311-312. 
[10] D.Ralescu and G.Adams, The fuzzy integral, J. Math. Anal. Appl. 75 (1980) 562-570.

[11] D.Revuz, Markov Chains (North-Holland, New York, 1975).

[12] W.E.Stein, Optimal stopping in a fuzzy environment, Fuzzy Sets and Systems 3 (1980) 253-259.

[13] M.Sugeno, Fuzzy measures and fuzzy integral : a survey in M.M.Gupta, G.N.Saridis and B.R.Gaines, Eds., Fuzzy Automata and Decision Processes (North-Holland, Amsterdam, 1977) 89-102.

[14] Y.Yoshida, M.Yasuda, J.Nakagami and M.Kurano, A potential of fuzzy relations with a linear structure: The contractive case, Fuzzy Sets and Systems 60 (1993) 283-294. 